\title{
Application of Fuzzy Mathematics in the Mold Simulation Software of Intelligent Assembly
}

\author{
Hua Sun and Wenbo Hao \\ Guidaojiaotong Polytechnic Institute, Shenyang 110023 China
}

\begin{abstract}
Taking the threaded hole in the virtual model as research object, According to the calibration method of absolute value reduction method, Based on fuzzy relationship matrix and fuzzy relationship matrix auto operation, make it meet the transitivity. According to the calculation results, the clustering analysis was carried out on the threaded hole. By hamming distance to establish the priorities ratio, constructs the fuzzy priorityratio matrix, implements the automatic assembly of the intelligent selection.
\end{abstract}

Keywords-fuzzy mathematics; matrix; clustering analysis; intelligent selection

\section{INTRODUCTION}

Automatic assembly of intelligent selection is a kind of artificial intelligence, AI (AI, short for Artificial Intelligence) is a branch of computer science, in the last century is known as one of the three most cutting-edge technology(artificial intelligence, energy technology, space technology). And is considered to be one of the three largest cutting-edge technology (artificial intelligence, nano science, genetic engineering) in this century,. For nearly 30 years in the field of artificial intelligence in many disciplines has been the rapid development of the application, and achieved fruitful results. Nowadays, artificial intelligence has become an independent branch of computer science both in theory and practice has become a system oneself. Article mainly depend on the calculation method of fuzzy mathematics, complete mold the parts in the automatic assembly of the intelligent selection.

\section{INTELLIGENT ASSEMBLY}

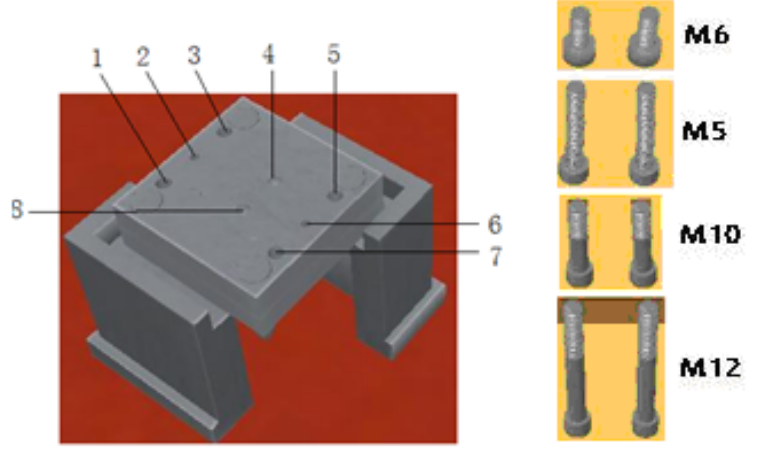

FIGURE I. MOLD INTELLIGENT ASSEMBLY

As shown in figure 1, We put the moving container and the moving container mould plate placed on the tooling in mould dismantling simulation software, and use screws to connect both. There are eight and eight screw threaded holes and the each screw must to connection with the corresponding threaded hole. How to let any one screw automatically to find the corresponding threaded hole? We first thread mouth for clustering analysis, and then preference analysis.

\section{CLUSTERING ANALYSIS}

Clustering analysis is a mathematical statistics research " Like attracts like " in a multivariate analysis method, which USES mathematical quantitatively determine the sample affinity-disaffinity relationship, so as to classify. ${ }^{[1]}$

Due to the fact itself in many cases with fuzziness, so the clustering analysis, introduced the method of fuzzy mathematics, can make the classification more down-to-earth. The fuzzy clustering analysis method can be roughly divided into two kinds: one is confused clustering method based on fuzzy relations, or called the system clustering analysis method. Another is called non-system clustering method, it is roughly divide the samples first, and then according to its classification for the optimal principle, after many iterations until classification more reasonable, this approach is also known as clustering method step by step.

After selected the statistical indicators, the method in fuzzy clustering analysis can be divided into the following three steps.

First, the representative point data standardization of statistical indicators, in order to analysis and comparison, this step is also known as normalization.

Calculate the following:

$x=\frac{x^{\prime}-x^{\prime}}{c}$

The $x^{\prime}$ as raw data, $\mathcal{X}^{\prime}$ are averages of the original data, c standard deviation to the original data.

If the standardized data compression to the closed interval $[0,1]$, the available extremum standardization formula

$$
x=\frac{x^{\prime}-x_{\text {min }}^{\prime}}{x_{\text {max }}^{\prime}-x_{\text {min }}^{\prime}}
$$


When $x^{\prime}=x^{\prime} \max , x^{\prime}=1$;

When $x^{\prime}=x^{\prime} \min , x^{\prime}=0$.

The second step is called calibration, namely the calculation measure similarity between objects are classified statistics $r_{i j}$ ( $\mathrm{i}=1,2, \ldots, \mathrm{n} \quad \mathrm{z}=1,2, \ldots, \mathrm{n}$; $\mathrm{n}$ for to be the number of classified objects), to determine similarity relation $\stackrel{R}{\sim}$ on the universe U.

$$
\underset{\sim}{R}=\left[\begin{array}{cccc}
r_{11} & r_{12} & \cdots & r_{1 n} \\
r_{21} & r_{21} & \cdots & r_{2 n} \\
\cdots & \cdots & \cdots & \cdots \\
r_{n 1} & r_{n 2} & \cdots & r_{n n}
\end{array}\right]
$$

There is a lot of method to calculate statistics $r_{i j}$, we choose absolutely reciprocal method

$$
r_{i j}= \begin{cases}1 & (i=j) \\ 1-c \sum_{k=1}^{m}\left|x_{i k}-x_{j k}\right| & (i \neq j)\end{cases}
$$

Selection of c should be appropriate, make $0 \leq r_{i j} \leq 1$.

Sometimes also can take please experienced expert grading, generally available centesimal system, and then divided by 100 for a quick closed interval of a decimal number, to avoid subjective, also can be used more than scoring average method to determine the $r_{i j}$ again

\section{$R$}

Step 3: clustering: $\stackrel{R}{\sim}$ must is a fuzzy equivalent to clustering. Have the relationship of reflexive, symmetric and transitivity called equivalence relation, and equivalent relation and decided to set a classification, we imitated the fuzzy equivalent relation can be defined. For the purpose of clear, we only consider can use fuzzy matrix expression of fuzzy relations $\stackrel{R}{\sim}$.

Domain $U$ is a limited collection is set theory, a fuzzy relationship $\underset{\sim}{R}=\left(r_{i j}\right)_{n \times n}$ set on a given $\mathrm{U}$, If it has:

(1) reflexivity $r_{i j}=1$

(2) symmetry $r_{i j}=r_{j i}(i, j=1,2, \cdots n)$

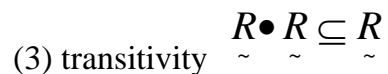

Called $\underset{\sim}{R}=\left(r_{i j}\right)_{n \times n}$ is a fuzzy hierarchy.
In this definition, visually reflexivity is on the diagonal $R$ elements of the matrix is 1 . Symmetry is $\sim$ is symmetric matrices, and the transitivity is difficult to directly see, need to

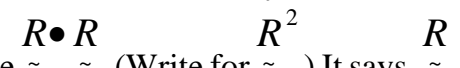
calculate $\stackrel{\sim}{\sim}$ (Write for $\sim$ ) It says $\stackrel{R}{\sim}$ and its synthesis, and then to see if it meet $\underset{\sim}{R \bullet} \underset{\sim}{R} \underset{\sim}{R}$.

For the theory of limited domain, is the product of matrix method

$$
\begin{aligned}
Q & =\left(r_{i k}\right)_{m \times l} \in F(U \times V), R=\left(r_{k j}\right)_{l \times n} \in F(V \times W) \\
Q \circ R & =S=\left(s_{i j}\right)_{m \times n} \in F(U \times W) \\
S_{i j} & =\underset{k=1}{\operatorname{l}}\left(q_{i k} \wedge r_{k j}\right) \quad(i=1,2, \cdots m \cdot j=1,2, \cdots n)
\end{aligned}
$$

Matrix synthesis also known as the product of matrix, it and common matrix multiplication operation process, just change the real ' + ' to ' $\vee$ ' ( if large value), the real ' $\bullet$ ' to ' $\wedge$ ' ( if small value).

We have eight threaded hole for classification, only consider the diameter of the hole. As shown in figure 1, unit is arranged according to clockwise direction.

Unit I ${ }^{X_{1}}: 10 \mathrm{~mm}$;Unit II ${ }^{X_{2}}: 5 \mathrm{~mm}$;Unit III ${ }^{X_{3}}: 12 \mathrm{~mm}$; Unit IV ${ }^{X_{4}}: 6 \mathrm{~mm} ; \mathrm{Unit}^{X_{5}}: 10 \mathrm{~mm}$; Unit VI ${ }^{X_{6}}: 5 \mathrm{~mm} ; \mathrm{Unit}$ VII $^{X_{7}}: 12 \mathrm{~mm}$;Unit VIII ${ }^{X_{8}}: 6 \mathrm{~mm}$.

Based on these data, according to the calibration method of absolute value reduction method;

$$
r_{i j}= \begin{cases}1 & (i=j) \\ 1-c \sum_{k=1}^{m}\left|x_{i k}-x_{j k}\right| & (i \neq j)\end{cases}
$$

Assignment $\quad c=0.05, m=1, i, j=1,2,3,4,5,6,7,8$;

So establishing fuzzy relationship matrix $R$ by calculation

$$
\underset{\sim}{R}=\left[\begin{array}{cccccccc}
1 & 0.75 & 0.9 & 0.8 & 1 & 0.75 & 0.9 & 0.8 \\
0.75 & 1 & 0.65 & 0.95 & 0.75 & 1 & 0.65 & 0.95 \\
0.9 & 0.65 & 1 & 0.7 & 0.9 & 0.65 & 1 & 0.7 \\
0.8 & 0.95 & 0.7 & 1 & 0.8 & 0.95 & 0.7 & 1 \\
1 & 0.75 & 0.9 & 0.8 & 1 & 0.75 & 0.9 & 0.8 \\
0.75 & 1 & 0.65 & 0.95 & 0.75 & 1 & 0.65 & 0.95 \\
0.9 & 0.65 & 1 & 0.7 & 0.9 & 0.65 & 1 & 0.7 \\
0.8 & 0.95 & 0.7 & 1 & 0.8 & 0.95 & 0.7 & 1
\end{array}\right]
$$

It is not hard to see, matrix is reflexive and symmetry, but not with transitivity. Therefore classified matrix can not directly. Square of $\mathrm{R}$; Then square of,

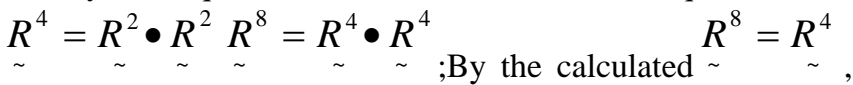


Therefore the selected $\stackrel{R^{4}}{\sim}$ as the fuzzy equivalent relation matrix, and Classified according to the above conclusion.

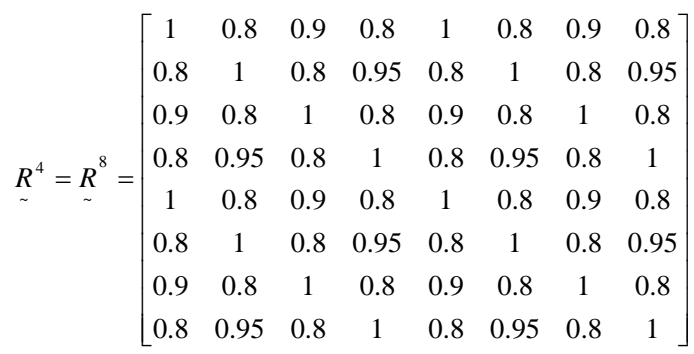

The values $\lambda=1$ the cutting matrix of $R^{4}$

$$
\underset{\sim}{R_{1}}=\left[\begin{array}{llllllll}
1 & 0 & 0 & 0 & 1 & 0 & 0 & 0 \\
0 & 1 & 0 & 0 & 0 & 1 & 0 & 0 \\
0 & 0 & 1 & 0 & 0 & 0 & 1 & 0 \\
0 & 0 & 0 & 1 & 0 & 0 & 0 & 1 \\
1 & 0 & 0 & 0 & 1 & 0 & 0 & 0 \\
0 & 1 & 0 & 0 & 0 & 1 & 0 & 0 \\
0 & 0 & 1 & 0 & 0 & 0 & 1 & 0 \\
0 & 0 & 0 & 1 & 0 & 0 & 0 & 1
\end{array}\right]
$$

Therefore, the thread hole can be divided into four categories: $\left\{x_{1}, x_{5}\right\} ;\left\{x_{2}, x_{6}\right\} ;\left\{x_{3}, x_{7}\right\} ;\left\{x_{4}, x_{8}\right\}$,

The diameter of the same thread hole is divided into a category, The calculation results accord with our expectations.

\section{INTELLIGENT SELECTION}

Due to the fuzzy mathematics method to simulate the brain thinking, so it has been widely used in artificial intelligence. ${ }^{[2]}$

Classification of screw as the table 1

TABLE 1. SSCREW CLASSIFICATION

\begin{tabular}{lllll}
\hline category & one & two & three & four \\
& & & & \\
\hline diameter & 6 & 5 & 10 & 12 \\
\hline
\end{tabular}

Now with screw $M 10 \times 10$ for fixed sample, comparing four types of threaded hole diameter and its similarity, Specific data are listed below.

By the hamming distance $d_{i}=\left|x_{k}-x_{i}\right|, d_{j}=\left|x_{k}-x_{j}\right|$; then establish the priorities ratio, is the similar priority ratio; $r_{i j}=\frac{d_{k i}}{d_{k i}+d_{k j}}, r_{j i}=1-r_{i j}$, constructs the fuzzy priority ratio matrix

$$
\underset{\sim}{R}=\left[\begin{array}{cccc}
1 & 0.56 & 0 & 0.33 \\
0.44 & 1 & 0 & 0.29 \\
1 & 1 & 1 & 1 \\
0.67 & 0.71 & 0 & 1
\end{array}\right]
$$

We can be found in the matrix $\mathrm{R}$

In the four types of threaded hole, the third type of threaded hole (M10) than the rest of the three categories is preferred. That is to say, in the four types of threaded hole, the third type of threaded hole in diameter with fixed sample $M 10 \times 10$ similar degree is better than the other three kinds of threaded hole, Screw will be priority and $10 \mathrm{~mm}$ diameter threaded hole for connection.

\section{CONCLUSION}

Article on the fuzzy clustering analysis method, implements the automatic assembly of the intelligent selection.

\section{REFERENCE}

[1] He Zhongxiong, Fuzzy sets and applications, Tianjin Science and Technology Press, 1983.

[2] Yang Lunbiao, The fuzzy mathematics principle and application, South China university of technology press,2005. 